\title{
Sunyaev-Zeldovich and Cosmic Microwave Background
}

\author{
Carlo Burigana**i \\ INAF-IASF Bologna, via Gobetti 101, I-40129 Bologna, Italy \\ E-mail: buriganaeiasfbo.inaf.it
}

Since its original formulation the Sunyaev-Zeldovich (SZ) effect has been recognized as a "powerful laboratory" for our comprehension of physical processes in cosmic structures and to derive crucial information on some general properties of the universe. After a discussion of the fundamental concepts and of some well established applications of the SZ effect towards galaxy clusters, I will focus on dedicated themes related to the SZ effect and other features in the cosmic microwave background (CMB) of particular interest in the view of the extremely high angular resolution observations achievable in the future with the Square Kilometre Array (SKA). SKA will allow the mapping of the thermal and density structure of clusters of galaxies at radio and centimetre bands with unprecedented resolution and sensitivity and with an extremely accurate control of extragalactic radio source contamination. The signatures from SZ effects and free-free emission at galactic scales and in the intergalactic medium probe the structure evolution at various cosmic times. The detection of these sources and their imaging at the high resolution and sensitivity achievable with SKA will greatly contribute to the comprehension of crucial cosmological and astrophysical aspects, as the physical conditions of early ionized halos, quasars and proto-galactic gas. The spectacular improvement in our understanding of the properties of extragalactic radio sources at very faint fluxes achievable with SKA will allow to accurately model their contribution to the diffuse radio background, greatly contributing to the interpretation of next generation of CMB spectrum experiments devoted to probe the thermal plasma history at early times.

First MCCT-SKADS Training School

September 23-29, 2007

Medicina, Bologna, Italy

\footnotetext{
*Speaker.

${ }^{\dagger}$ Dipartimento di Fisica, Università degli Studi di Ferrara, via Saragat 1, I-44100 Ferrara, Italy
} 


\section{Introduction}

The thermal plasma in the intergalactic and intracluster medium and at galactic scales leaves imprints on the cosmic microwave background (CMB) through the Thomson scattering of CMB photons on hot electrons (Sunyaev-Zeldovich effect) and the free-free emission. Since its original formulation the Sunyaev-Zeldovich (SZ) effect has been recognized as a "powerful laboratory" for our comprehension of physical processes in cosmic structures and to derive crucial information on some general properties of the universe. The characterization of the free-free emission distortion in many astrophysical and cosmological contexts may suffer of an intrinsic uncertainty because of the relatively strong dependence of this effect on matter density, typically implying a certain model dependence in the estimates of its amplitude. In spite of this, the theoretical predictions of several models could be probed through new measures at radio and centimetre wavelengths.

After a discussion of the fundamental concepts (Sect. 2) and of some well established applications (Sect. 3) of the SZ effect towards galaxy clusters, I focus on dedicated themes related to the SZ effect and other features in the CMB of particular interest in the view of the extremely high angular resolution observations achievable in the future with the Square Kilometre Array (SKA) (Sect. 3.3 and Sect. 4). In fact, although not specifically devoted to CMB studies, because of its high resolution and the limited high frequency coverage, right the extreme sensitivity and resolution of SKA may be fruitfully used for a detailed mapping of these effects on dedicated sky areas. The SKA highest frequencies $(\simeq 20-30 \mathrm{GHz})$, that will be implemented during the third phase of the project [39], are, of course, the most advantageous in particular for the study of SZ effects because of the steeper decrease of the synchrotron radio emission with the frequency.

For sake of conciseness, I avoid in this work to include figures as well as to repeat a complete reporting of the derivations of some fundamental equations, focussing, on the contrary, to their physical meaning. They are in fact available in the presentation uploaded at the School web site ${ }^{1}$ (you can also ask the presentation to the author). I will often refer in the following to the appropriate presentation slide number.

\section{Kompaneets equation and imprints on the CMB}

Under general condition, the evolution of CMB photon occupation number, $\eta(v)$ (at the frequency $v$ ), which determines the CMB photon energy density $\varepsilon_{\mathrm{CMB}}=8 \pi h_{P} / c^{3} \int \eta(v) v^{3} d v$, is described by the kinetic (Boltzmann) equation; here $h_{P}$ is the Planck constant and $c$ the speed of light. Various physical processes can contribute to the evolution of $\eta$. In many cosmological and astrophysical contexts the Compton scattering is the most efficient interaction in the plasma able to exchange energy between (baryonic) matter and radiation. From fundamental electromagnetism concepts one can derive the Compton cross-section and the energy exchange between electron and photon in a single scattering (see e.g. [80]; see slides 4-6). In the Thomson limit $\left(\varepsilon / m_{e} c^{2} \ll 1\right.$, where $\varepsilon$ is the photon energy before scattering and $m_{e}$ the electron mass; $m_{e} c^{2} \simeq 511 \mathrm{keV}$ ) the photon energy $\varepsilon_{1}$ after scattering is not so different from the original one and the differential crosssection is almost isotropic. From the energy-momentum conservation law in the electron rest frame it is possible to derive the photon dimensionless energy, $x_{1}=h_{P} v_{1} / k_{B} T_{e}$, after scattering as function

\footnotetext{
${ }^{1}$ http://www.ira.inaf.it/ sschool_loc/presentations/PDF/Carlo_Burigana.pdf
} 
of the photon dimensionless energy, $x$, before scattering, of the electron energy and momentum, and of the photon directions before and after scattering; here $T_{e}$ is the electron temperature and $k_{B}$ the Boltzmann constant. In general, in the electron rest frame, $x_{1}<x$, or, in other words, the photon gives a fraction of its original energy to the electron. The conclusion is different passing to the laboratory frame: in this case, because of Doppler effect, the photon energy can be increased of decreased after the scattering, depending on the relevant electron and photon motion directions. The number of scatterings per unit time depends on the electron density, $n_{e}$, and on the photon $(c \vec{n})$ and electron $(\vec{v})$ velocities

$$
\frac{d N}{d t}=\sigma_{T} n_{e} c[1-(\vec{v} / c) \cdot \vec{n}] ;
$$

here $\sigma_{T}$ is the Thomson cross-section and $t$ is the time.

Averaging over an ensamble of electrons at a temperature $T_{e}$ and using the semi-isotropy of scattering holding in the Thomson limit, the average change of the photon energy per unit time due to the Doppler effect can be expressed by (see slide 6)

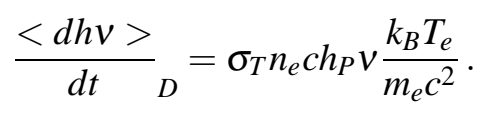

The kinetic equation describes as $\eta(v)$ changes because of the contribution from photons at different frequencies and the migrations of photons from the frequency $v$ to frequencies different from $v$ because of Compton scattering (see slide 8). It is general but at the same time very difficult to solve. For this reason, various approximations of it have been proposed in the literature according to the assumptions applicable to the considered astrophysical or cosmological context. The most famous approximation has been derived by Kompaneets many years before its publication [51]. It is a second order approximation of the kinetic equation in the dimensionless energy difference $\Delta=x_{1}-x$ in the Thomson limit for the differential cross-section and for a Maxwellian distribution of electrons (see slide 8). These two assumptions greatly simplify the second order serie expansion allowing to identify two contributions to the time evolution of the photon occupation number, $\frac{\partial \eta}{\partial t}$ : a secular shift term, $\propto \Delta$, and a random walk term, $\propto \Delta^{2}$. Thanks to the semi-isotropy of the Thomson differential cross-section the latter term can be easily computed directly (see slide 9 ). The derivation of the secular shift term can instead be performed using the fundamental property of scattering, i.e. the photon number conservation. It sets a constraint on the photon flux on a spherical surface of the phase space at a given frequency, $j(x)$, equivalent to the relation

$$
\frac{1}{x^{2}} \frac{\partial j(x)}{\partial x}=\frac{\partial \eta}{\partial t}
$$

Since no products of the first and second derivative of $\eta$ with respect to $x$ appear in $\frac{\partial \eta}{\partial t}$, then $\frac{\partial \eta}{\partial x}$ can appear only linearly in the function $j$. In addition, at thermal equilibrium, when the CMB spectrum is a blackbody (BB), $j=0=\frac{\partial \eta}{\partial t}$. This sets the form of $j(x)$ to be a product of a function of $\eta$ by an unknown function $g(x)$. $\frac{\partial \eta}{\partial t}$ can be formally expressed by Eq. (2.3) and by the above second order serie expansion. Therefore, when the random walk term is explicited, their equality and, in particular, the equality between the coefficients in front of the first and second derivative of $\eta$ with respect to $x$ allows to determine the form of the function $\mathrm{g}(\mathrm{x})$ and then the expression of the secular shift term (see slide 10). One then arrives to the Kompaneets equation (for the scattering 
Compton term alone). In cosmological context, it is important to note that the dimensionless photon energy $x=h_{P} v / k_{B} T_{e}$ is in general not redshift invariant, depending on the redshift dependence of $T_{e}$. It is then more advantageous to introduce a different dimensionless photon energy defined by $x=h_{P} v /\left[k_{B} T_{0}(1+z)\right]$ where, independently of the exact form of the photon occupation number at the present time $t_{0}, a_{B B} T_{0}^{4}$ gives the present $\mathrm{CMB}$ energy density, $\varepsilon_{C M B, 0}, a_{B B}$ being the $\mathrm{BB}$ constant. It is useful to introduce a "nominal" $\mathrm{CMB}$ energy density $\varepsilon_{\mathrm{CMB}}=a_{B B} T_{\mathrm{CMB}}^{4} \simeq 4.2 \times$ $10^{-13}(1+z)^{4} \mathrm{erg} \mathrm{cm}^{-3}$, where $T_{\mathrm{CMB}}=T_{0}(1+z)$ is a "nominal" CMB temperature ${ }^{2}$. In this way, $x$ is redshift invariant (since also $v$ scales as $1+z$ during cosmic expansion). Adopting this last choice of $x$, the Kompaneets equation can be written as

$$
\frac{\partial \eta}{\partial t}=\left(\frac{\partial \eta}{\partial t}\right)_{C}=\frac{1}{\phi} \frac{1}{t_{C}} \frac{1}{x^{2}} \frac{\partial}{\partial x}\left[x^{4}\left[\phi \frac{\partial \eta}{\partial x}+\eta(1+\eta)\right]\right] ;
$$

here $t_{C}=m_{e} c^{2} /\left[k_{B} T_{e}\left(n_{e} \sigma_{T} c\right)\right]$ is the gas cooling time by Thomson scattering and $\phi=T_{e} /\left[T_{0}(1+z)\right]$ is a dimensionless electron temperature. Three terms can be identified in Eq. (2.4): ordinary and induced Compton scattering, respectively proportional to $\eta$ and $\eta^{2}$, that tend to move photons towards low frequencies and inverse Compton scattering, the only one involving the second derivative of $\eta$, that tends to move photons towards high frequencies [22]. It is important to remember that Eq. (2.4) needs to be coupled with the appropriate (differential equation describing the) time evolution of the electron temperature (see slides 7 and 24) in order to avoid to introduce significant errors [11] in its solution ${ }^{3}$.

At this point it is important to remember the limits of validity of this equation (see slide 12): a large number of scatterings is assumed (otherwise it has no meaning to compute averages); non relativistic electrons and relatively soft photons are assumed; the photon occupation number shape should be smooth (otherwise $\frac{\partial \eta}{\partial x}$ could be much larger than $\eta$, and so on for higher order derivatives, and a serie expansion is no longer adequate). Finally, at least in this form, the Kompaneets equation assumes isotropic distributions of electrons and photons.

Note that, in general, for a Bose-Einstein (and, obviously, also for the particular case of a Planckian) distribution $\frac{\partial \eta}{\partial t}=0$ (see slide 15 for the fundamental properties of these distributions): in other words, if there is enough time to allow a sufficient number of scatterings, under the action of Compton scattering alone the photon occupation number tends towards a Bose-Einstein (BE) distribution which then represents the (general) kinetic equilibrium solution. Typically, for CMB photons, this condition is satisfied at high redshifts at least at high frequencies, where other radiative processes are not so important. The chemical potential ${ }^{4} \mu$ of the BE distribution is related to the fractional energy exchange occurred between matter and radiation associated to the dissipation process at the origin of the deviation from the full equilibrium BB spectrum [90, 22]:

$$
\mu \simeq 1.4 \Delta \varepsilon / \varepsilon_{i} ;
$$

the case of small distortions (or small energy exchanges $\Delta \varepsilon / \varepsilon_{i} \ll 1$, at least in the case of dissipation processes with negligible photon production) is considered here for simplicity; $\varepsilon_{i}$ is the CMB

\footnotetext{
2"Nominal" refers here to the case in which the CMB energy density at any epoch scales simply as $(1+z)^{4}$, i.e. in the absence of energy exchanges on the cosmic plasma.

${ }^{3}$ The dependence of the Compton equilibrium electron temperature $[71,100]$ on integrals of $\eta$ increases the computational complexity of the (numerical) solution of Eq. (2.4) [11].

${ }^{4}$ In this context, the dimensionless chemical potential is typically used.
} 
energy density before energy exchange. This result can be easily derived comparing the photon energy density and number density of the original BB spectrum and of the distorted BE spectrum. It is important to note that, observationally, COBE/FIRAS data set stringent limits to the amplitude of spectral distortions at redshifts lower than $\approx 10^{6}$, while these limits are significantly relaxed at earlier epochs $[31,81]$ (see slides 28-29 and 31). At $z \gtrsim z_{\text {therm }}, z_{\text {therm }}$ being the thermalization redshift (see slide 30) [11], the limits on $\Delta \varepsilon / \varepsilon_{i}$ are set by the cosmological nucleosynthesis theory, that, in order to explain chemical abundances, implies an early comoving photon energy density not too far from the present one.

A very different solution can be found under two different assumptions. If $\phi \gg 1$ (hot electrons) the inverse Compton term dominates over ordinary and induced Compton terms. Neglecting these two terms, the Kompaneets equation becomes equivalent to the heat diffusion equation (see e.g. [95]) and admits an exact analytical solution described by a superposition of blackbodies $[101,102]$. Note that (see slide 16) for small deviations from an initial BB spectrum the Kompaneets equation is equivalent to the inverse Compton dominated case except for a further factor $\left(1-\phi_{i} / \phi\right)$ where $\phi_{i}$ is the initial electron and (BB) radiation dimensionless temperature (corresponding to a physical temperature $T_{i}$ ). A simple solution, the so-called Comptonization solution, can be found in this case:

$$
\eta(x, \tau) \simeq \eta_{i}+u \frac{x / \phi_{i} \exp \left(x / \phi_{i}\right)}{\left[\exp \left(x / \phi_{i}\right)-1\right]^{2}}\left(\frac{x / \phi_{i}}{\tanh \left(x / 2 \phi_{i}\right)}-4\right),
$$

where $\eta_{i}$ is the initial distribution function and $u$ is the (evolving) Comptonization parameter (see e.g. [12])

$$
u(t)=u(z)=\int_{t_{i}}^{t} \frac{\phi-\phi_{i}}{\phi} \frac{d t}{t_{C}}=\int_{1+z}^{1+z_{i}}\left(\phi-\phi_{i}\right)\left(\frac{k_{B} T_{r}}{m_{e} c^{2}}\right) n_{e} \sigma_{T} t_{\exp } \frac{d\left(1+z^{\prime}\right)}{1+z^{\prime}} ;
$$

here $t_{\text {exp }}=a /(d a / d t)$ is the cosmic expansion time. Obviously, the case of hot electrons is directly obtained from this equation in the limit $\phi \gg \phi_{i}$. This formula is an excellent approximation of the exact solution. By integrating $u$ over the whole relevant energy dissipation phase one gets the "usual" Comptonization parameter related to the whole fractional energy exchange by the well known expression [101]

$$
u \simeq(1 / 4) \Delta \varepsilon / \varepsilon_{i}
$$

again, the case of small distortions is considered for simplicity in the above equation. This result can be easily derived considering how the photon energy density changes because of the evolution of $\eta$ (see slide 13).

Note that in the Rayleigh-Jeans (RJ) region Eq. (2.6) predicts a lowering of the photon occupation number (see slide 16) and of the brigthness temperature (see slides 17). This effect decreases at increasing frequency up to $217 \mathrm{GHz}$ where it vanishes. Then, for a further frequency increasing the formula predicts an increasing signal fractional excess with respect to the original BB. In other words, inverse Compton scattering tends to create a photon distribution with more (respec. less) high (respec. low) frequency photons than those of the original BB. In cosmological context this kind of departure from a BB (i.e. of CMB spectral distortion) is expected at relatively late epochs (at early ones there is time enough to achieve the kinetic equilibrium) in the presence of a diffuse medium at temperature larger than that of the $\mathrm{CMB}\left(T_{\text {matter }}=T_{e}>T_{0}(1+z)\right.$; see slides $\left.32-33\right)$, 
but also under many physical conditions (see e.g. [22]). Note that, at least in principle, a cooling process $\left(T_{\text {matter }}=T_{e}<T_{0}(1+z)\right.$ ) could produce a negative Comptonization distortion [88] (see slides 32 and 36). A remarkable example ${ }^{5}$ of (positive) Comptonization distortion is that associated to the cosmological reionization that affects the CMB both in anisotropies at large and small scales and in the spectrum (see slide 81; see [21]; see also Sect. 4.3). For instance, the reionization redshift (see slide 82) identified by the NASA Wilkinson Microwave Anisotropy Probe ${ }^{6}$ (WMAP) satellite 3-yr data is consistent with minimal reionization models [82] predicting a Comptonization distortion with $\Delta \varepsilon / \varepsilon_{i} \simeq 4 u \approx 4-7 \times 10^{-7}[15]$.

At low frequencies, other radiative processes can not be neglected and the corresponding terms have to be included in the (complete) Kompaneets equation:

$$
\frac{\partial \eta}{\partial t}=\left(\frac{\partial \eta}{\partial t}\right)_{\Lambda}+\left(\frac{\partial \eta}{\partial t}\right)_{\Gamma}=\sum \Lambda_{i}+\sum \Gamma_{i}
$$

here $\sum \Lambda_{i}$ takes into account processes that does not change the photon number (typically, the Compton scattering) and $\sum \Gamma_{i}$ takes into account photon production/absorption processes (see slides 20-22).

Three mechanisms certainly operate in the cosmic plasma: bremsstrahlung (BR) or free-free (FF) [46, 80], double (or radiative) Compton (DC) [34], and cyclotron (CE) emission. Recently, [103] demonstrated that, for realistic values of cosmic magnetic field, the cyclotron process never plays an important role for (global) CMB spectral distortions when ordinary and stimulated emission and absorption are properly taken into account and CMB realistic distorted spectra, as discussed in the following, are considered. In fact, the cyclotron term may be significant, in the case of deviations of $\eta$ from the BB distribution at the electron temperature, only at very long wavelengths, corresponding to the cyclotron frequency, where, during the formation of a spectral distortion, FF and DC are able to keep $\eta$ extremely close to the BB equilibrium. These processes are in fact very efficient at long wavelengths because of their $\approx x^{-3}$ dependence. So, at high redshifts the $\mathrm{BE}$ solution needs to be modified introducing a frequency dependence in the chemical potential, $\mu=\mu(x)$, which vanishes at very long wavelengths [90,23]. The resulting spectrum (see slide 25) has then a minimum in equivalent thermodynamic temperature ${ }^{7}, T_{t h}$, at a wavelength depending on the baryon density [23, 11]. Note that DC (respec. FF) is more important that FF (respec. DC) at high (respec. low) redshifts $\left(z\right.$ higher (respec. lower) than $\approx 10^{5}$ ). In general, if there is enough time, the long wavelength photon production by DC and FF combined to the shifting of

\footnotetext{
${ }^{5}$ In the past, before of its explanation in terms of integrated contribution by discrete sources, the cosmic X-ray background (see slide 34) has been interpreted in terms of bremsstrahlung radiation by hot diffuse medium. In this framework, a substantial CMB Comptonization distortion was predicted, but right the stringent upper limit to this kind of CMB distortion posed by COBE/FIRAS sets the most stringent upper limit to the fraction of cosmic X-ray background that could be produced by diffuse hot gas (see slide 35).

${ }^{6} \mathrm{http}: / /$ lambda.gsfc.nasa.gov/product/map/current/

${ }^{7} T_{t h}$ is defined as the temperature of a BB with the same $\eta(v)$ as that under consideration, i.e. such that $\eta(v)=$ $1 /\left[\exp \left(h_{P} v /\left(k_{B} T_{t h}(v)\right)\right)-1\right]$. Only for $\eta=\eta_{B B}, T_{t h}$ is independent of $v$, while for an arbitrary photon occupation number $T_{t h}$ is frequency dependent, $T_{t h}=T_{t h}(v)$. In the following $T_{t h}$ will be called also brightness temperature and denoted by $T_{b r}$. Note that the brightness temperature, $T_{b r}$, is often used in the literature also with the meaning of antenna temperature $T_{\text {ant }}$, defined as the temperature of a BB with the same $\eta(v)$ as that under consideration but in the RJ limit, i.e. $\eta(v)=1 /\left[h_{P} v /\left(k_{B} T_{a n t}(v)\right]\right.$. Obviously, $T_{a n t} \simeq T_{t h}$ in the RJ limit.
} 
produced photon to higher frequencies thanks to Compton scattering tends to reduce the amplitude of a possible early spectral distortion, being at limit able to fully re-establish a BB (see slides 28-31), i.e. to thermalize the CMB spectrum [11].

The BE and Comptonization like distortions discussed above (properly modified with respect to the pure $\mathrm{BE}$ and Comptonization distributions because of the effect of DC and FF) describe distorted spectra at early and late epochs, respectively (see slide 18). For processing possibly occurred at intermediate epochs a distorted spectral shape intermediate between these two cases is predicted (see slide 26). In particular, for processes at relatively late epochs a plateau (in $T_{t h}$ ) is produced in the RJ region, with a brigtness temperature decrement related also to the process epoch $^{8}$ [12] (see slide 26, panel b).

At relatively low redshifts, FF, instead of thermalizing the spectrum to a BB at the electron temperature (this is achieved in any case at extremely low frequencies), can produce the so-called free-free (low frequency) distortion [12]. It is easy to verify (see slide 23) that, in the RJ region, the $x^{-3}$ dependence of FF and the weak frequency dependence of the FF Gaunt factor implies a fractional increase of the equivalent thermodynamic temperature almost proportional to $x^{-2}$, characterized in amplitude by a simple parameter, the so-called FF distortion parameter, $y_{B}$. A remarkable excess can be produced at centimetre and decimetre wavelengths (see slide 33) - or, in principle, a decrement in the case of cooling processes (i.e. negative distortions; see slide 36 ).

\section{Thermal and kinetic SZ effect towards galaxy clusters}

The Comptonization solution described in the previous section is at the basis of the well known (thermal) SZ effect. The form of Eqs. (2.6) and (2.7) are appropriate to the case of CMB global distortions, but it easy to rewrite these equations in the case of the interaction of CMB photon with hot gas in a cluster of galaxies (or in a primeval halo or, at smaller scales, in the hot medium surrounding a galaxy). It is enough to replace the integral in $n_{e} c d t$ over the relevant cosmic time with an integral in $n_{e} d l$ over the cluster along the line of sight in Eq. (2.7) (see slide 37).

The SZ effect due to the scattering of CMB photons with hot electrons in galaxies and clusters of galaxies is typically expressed in terms of a frequency dependent change in the CMB brightness (see slides 39-40). If the hot electron gas is globally at rest with respect to the observer, only the thermal SZ effect [91] (see also [78]) will be present; differently, a bulk peculiar motion, $V_{r}$, of the hot electron gas produces a kinetic SZ effect (see slide 38). In the RJ region the first effect produces a decrement of the surface brightness, $\Delta I_{t h}$, towards the cluster. The second effect produces either a decrement or an increment, $\Delta I_{k}$, depending on the direction of the cluster velocity with respect to the observer. Neglecting relativistic corrections:

$$
\Delta I_{t h}=I_{0} y g(x)
$$

and

$$
\Delta I_{k}=-I_{0}\left(V_{r} / c\right) \tau_{e} h(x),
$$

\footnotetext{
${ }^{8}$ It can be formally derived with a further approximation of the Kompaneets equation in terms of small deviations from the Comptonization spectrum.
} 
where $I_{0}=\left(2 h_{P} / c^{2}\right)\left(k_{B} T_{C M B} / h_{P}\right)^{3}$. Here

$$
\tau_{e}=\int n_{e} \sigma_{T} d l
$$

and

$$
y=\int\left(k_{B} T_{e} / m_{e} c^{2}\right) n_{e} \sigma_{T} d l
$$

are respectively the Thomson optical depth and the Comptonization parameter ${ }^{9}$ [101] integrated over the cluster along the line of sight and

$$
\begin{gathered}
h(x)=x^{4} e^{x} /\left(e^{x}-1\right)^{2} \\
g(x)=h(x)\left[x\left(e^{x}+1\right) /\left(e^{x}-1\right)-4\right] .
\end{gathered}
$$

The two effects have a different frequency dependence that in principle allows their separation through multi-frequency observations (see slides 41-42). SKA observations in the RJ regime (where $h(x) \sim g(x) \rightarrow x^{2}$ ) can be combined with millimetric observations. In particular, $g(x) \simeq 0$ and $h(x)$ is maximum at $\sim 217 \mathrm{GHz}$, a frequency that, exactly for this reason, is typically included in SZ multifrequency observations at millimetre wavelengths. Accurate measures of the kinetic SZ effect can provide a crucial cosmological information on cluster velocity field (see e.g. [5] for results achieved with SuZIE II).

\subsection{Treatments of the thermal SZ effect beyond the Kompaneets approximation}

The above treatment of the SZ effect is based on the Kompaneets equation and obvioulsy assumes the same hypotheses (or work conditions).

Two main lines have been followed to overcome the limits of validity of this formalism. From one side one can search for solutions more appropriate to the case of a small number of scatterings between CMB photons and electrons, possibly including (at least to a given order) the KleinNishina correction to the classical Thomson scattering cross-section [99, 29]. From the other side, it is interesting to search for approximations of the kinetic equation not only up to the second order but up to a higher order, necessary to treat the relativistic case [42]. These approaches are physically motivated on the basis of the high electron temperatures often achieved in clusters of galaxies ${ }^{10}$ and/or by small values of the optical depth (see Eq. (3.3)).

The general properties of the solutions found using these treatments are not so different from those predicted by the original SZ treatment. See slide 35 for an application of the method described in [29]: note the slightly steeper behaviour at high frequencies found in this case. See slide 44 for a comparison between non-relativistic and relativistic approximations [79]: note how the difference, clearly appreciable at high frequencies, is not so critical in the RJ region (relevant for SKA). Note also that only a small shift of the frequency of vanishing effect with respect to the non relativistic case $(217 \mathrm{GHz})$ is predicted.

\footnotetext{
${ }^{9}$ I prefer to use here the symbol $u$ for the "global" Comptonization parameter of a CMB distorted spectrum and the symbol $y$ for the "local" Comptonization parameter characterizing the SZ effect.

${ }^{10}$ Or for a diffuse very hot gas - see also footnote 3.
} 


\subsection{Some cosmological applications of SZ effect towards galaxy clusters}

The exploitation of the cosmological applications and implications of the SZ effect towards galaxy clusters has received a large attention from both the theoretical and the observational point of view. The reader could refer for example to [58] (and references therein) for exhaustive discussions (see slide 57). I report here on three interesting examples.

\subsubsection{Hubble constant determination}

As well known, one of the most remarkable difficulties in the accurate measure of the Hubble constant, $H_{0}=[(d a / d t) / a]_{0}$ (see e.g. [97]), which determines the universe expansion rate at the present time, has been represented for a long time by the necessity to precisely calibrate various kinds of "standard candles". So, some attempts have been dedicated to find methods able to measure $H_{0}$ (as well as other cosmological parameters) circumventing this (or other) difficulty(ies). An interesting solution has been proposed in the ' 70 by [19, 36, 86, 20] (see also [67]). It involves the combination of X-ray and radio observations towards cluster of galaxies (see slides 46-48). The different dependence of bremsstrahlung, responsible of the cluster X-ray emission, and of SZ effect (namely the decrement of the brigthness temperature in the RJ region) towards the cluster on the relevant physical and geometrical parameters allows to derive a simple expression for the cluster luminosity distance in terms of ratio between the square of the brigthness temperature decrement in the RJ region and the X-ray brightness, of cluster electron temperature, cluster (angular) core radius, and cluster redshift. Note that these quantities are, in principle, measurable. Therefore, a comparison with the usual Hubble expression for the luminosity distance (see slide 48) allows to directly derive $H_{0}$. A certain degradation of the reliability of this method comes from the complexity of the geometrical and physical properties of clusters (see e.g. slide 49). For example, different baryonic physics results into noticeable differences in the surface distributions and in the profiles of the X-ray brightness, projected temperature, and Comptonization parameter, particularly for the inner cluster regions [62] (see slide 50). Another remarkable difficulty of the method and, in general, of cluster modelling, is represented by the presence of radio sources in the cluster that, with their surface density distribution different from that of control fields, can affect the signal due to the "pure" SZ effect, a problem recently addressed by [55] (see slides 51-52).

Only recently, accumulating an increasing number of clusters, this method to measure $H_{0}$ starts to provide reliable results [18]. In spite of the above difficulties, the large increasing of number of clusters that will be possible to consider in the next future (see Sect. 3.3) and the continuous improvement in the observation and physical modelling of clusters, will make this method very interesting at least for an almost independent cross-check of the results achievable through other measurements, first of all from the CMB anisotropy.

\subsubsection{Determination of cosmological parameters through statistical approaches}

The Hubble constant is only one of the parameters that determine the "background"11 evolution of the universe, while other parameters characterize the dishomogeneity properties of the

\footnotetext{
11 "Background" is used here to denote the global properties of a ("virtual") perfectly isotropic and homogeneous universe with all ("virtual") galaxies following the pure Hubble expansion flow.
} 
universe related to the formation and evolution of cosmic structures. On the other hand, the "background" evolution of the universe plays a crucial role also for the structure formation process. Therefore, the study of the correlation properties of such dishomogeneities provides information on the cosmological parameters originally introduced to characterize the "background" properties of the universe.

Likely, the most useful (and used) statistical estimator of such dishomogeneities projected on the celestial sphere is their angular power spectrum, $C_{\ell}$, as function of the multipole ${ }^{12}, \ell$, that, observationally, is a simple transform of the angular (auto)correlation function [69]. For Gaussian distributions, they completely describe the statistical properties of the fluctuation field. From the astrophysical side, $C_{\ell}$ is related to the redshift dependent mass function and profile of clusters. Different models for the mass function and for characterizing the physical profile of clusters imply different shape and amplitude of $C_{\ell}$ (see slide 53). In particular, it takes memory of some cosmological parameters, such as the density contrast (i.e. the linear theory amplitude of matter fluctuations), $\sigma_{8}$, at a characteristic scale (typically, $8 h^{-1} \mathrm{Mpc}$ ) and the global matter density (see $[79,2]$ and reference therein); here $h$ is the Hubble constant in units of $100 \mathrm{~km} \mathrm{~s}^{-1} \mathrm{Mpc}^{-1}$. So, the comparison between the observed and the theoretically predicted cluster distribution allows to determine such parameters or can be used with other cosmological data to remove the degeneracy in the determination of some cosmological parameters, existing using a single kind of observables (see e.g. $[8,9,28]$ ), such as the cosmological constant (or dark energy) and the matter (baryonic plus dark matter) density parameters (see slides 54-55).

\subsubsection{The SZ effect as a foreground for CMB studies}

Recently, a spectacular improvement in the assessment of cosmological models and in the determination of the cosmological parameters has been achieved thanks to the precise mapping of $\mathrm{CMB}$ anisotropies through balloon-borne, ground-based, and, in particular, space experiments (see e.g. [6], and references therein, for a review on pre-WMAP observational results). WMAP measured CMB anisotropies on the whole sky in both total intensity (or temperature, see 1-yr results [4]) and polarization (see the 3-yr results [43, 66]) Stokes parameters. A substantial improvement is expected in the next years from the forthcoming ESA Planck $^{13}$ satellite $[53,76,75]$.

The SZ effect from the ensamble of the clusters at various redshifts and sky positions constitutes a source of foreground for these projects, particularly remarkable at small scales (or large $\ell$ ). The SZ effects towards the brightest clusters can be directly identified on the CMB anisotropy maps and can be subtracted almost accurately from the fluctuation map during data analysis. On the contrary, those below a certain threshold can not be extracted from the fluctation field and contribute to the overall angular power spectrum (see slide 56) $[38,27]$. Therefore, the contribution of SZ effect from clusters to the overall $C_{\ell}$ needs to be accurately modelled and subtracted to extract the $C_{\ell}$ corresponding primary CMB fluctuations. These tasks, already carried out in the WMAP data analysis, are more relevant, and complex, in the case of the next Planck data analysis since it is aimed at measuring the $\mathrm{CMB} C_{\ell}$ at significantly higher multipoles. Clearly, the Planck wide frequency coverage, from $30 \mathrm{GHz}$ to $857 \mathrm{GHz}$, i.e. at frequencies both below and above $\simeq 217 \mathrm{GHz}$ where the

\footnotetext{
${ }^{12}$ The multipole $\ell$ is almost inversely proportional to the angular scale, $\ell \simeq 180 / \theta\left(^{\circ}\right)$.

${ }^{13} \mathrm{http}: / /$ www.rssd.esa.int/planck
} 
SZ effect vanishes, will allow to detect both the decrement and the excess of the CMB brightness temperature. This will greatly help the identification of this source of foreground, providing at the same time a rich information on cluster physics (see also the next section).

\subsection{SKA perspectives}

Many thousands of galaxy clusters can be identified on the whole sky by XMM $\left(\sim 10^{3}\right)$, Planck $(\sim 5000)$, and SDSS $\left(\sim 510^{5}\right)$. The typical angular sizes of galaxy clusters range from $\sim \operatorname{arcmin}$ to few tens of arcmin.

The SKA sensitivity and resolution mainly depends on the used array collecting area (see slides 60-63). By considering a frequency band of $\simeq 4 \mathrm{GHz}$ at $20 \mathrm{GHz}$, the whole instrument collecting area will allow to reach a (rms) sensitivity of $\simeq 40 \mathrm{nJy}$ in one hour of integration with an angular resolution of $\simeq 1$ mas (considering $\simeq 3000 \mathrm{~km}$ maximum baseline). By using only about $50 \%$ of the collecting area within $\simeq 5 \mathrm{~km}$, the (rms) sensitivity in one hour of integration is $\simeq 80 \mathrm{nJy}$ with a resolution of $\simeq 0.6^{\prime \prime}[39,13]$.

The major role on the study of the SZ effects towards galaxy clusters will be obviously played by dedicated telescopes operating at $\simeq$ arcmin resolutions with frequency coverages up to $\simeq$ millimetric wavelengths [44] and, at higher resolutions, by $\mathrm{ALMA}^{14}$. On the other hand, with the $50 \%$ of the SKA collecting area it will be possible to accurately map the SZ effect [89] of each considered cluster, particularly at moderately high redshifts, with the unprecedented precise subtraction of discrete radio sources achievable with SKA. The combination with accurate X-ray images of the cluster bremsstrahlung emission will allow to accurately map the thermal and density structure of the gas in galaxy clusters, thanks to the different dependences of these two effects on matter density and temperature. Of particular interest in this respect will be the observations expected by the wide field imager (WFI) on board the X-ray Evolving Universe Spectroscopy (XEUS) satellite ${ }^{15}$, recently selected by ESA as new candidate for possible future scientific missions ${ }^{16}$, designed to reach a resolution of $0.25^{\prime \prime}$ on a FOV of $5^{\prime}-10^{\prime}$ (see slides 64-67).

As mentioned above, it is also possible to study the SZ effect (both thermal and kinetic) from clusters in a statistical sense, namely through its contribution to the angular power spectrum of the CMB secondary anisotropies (see slides 68-70; see also Appendix A in [13]). This topic has been investigated in several papers (see, e.g., [65, 96, 33, 87, 26, 52]). At sub-arcmin scales (i.e. at multipoles $\ell \gtrsim 10^{4}$ ) secondary anisotropies from thermal (more important at $\ell \lesssim$ few $\times 10^{4}$ ) and kinetic (more important at $\ell \gtrsim$ few $\times 10^{4}$ ) SZ effect dominate over CMB primary anisotropy (see slide 78) whose power significantly decreases at multipoles $\ell \gtrsim 10^{3}$ because of photon diffusion (Silk damping effect [85]). Their angular power spectrum at $\ell \sim 10^{4}-10^{5}\left(\approx 10^{-12}-10^{-13}\right.$ in terms of dimensionless $C_{\ell} \ell(2 \ell+1) / 4 \pi$ ) could be in principle investigated with the sensitivity achievable with SKA (see slides 71-77; see [47] and Appendix A in [13]). On the other hand, at the SKA resolution and sensitivity the contribution to fluctuations from foreground sources (both diffuse radio emission, SZ effects, and free-free emitters) at galaxy scales likely dominates over the SZ effect from clusters (see slides 79-80).

\footnotetext{
${ }^{14} \mathrm{http}: / /$ www.alma.cl/

${ }^{15} \mathrm{http}: / /$ sci.esa.int/science-e/www/area/index.cfm?fareaid=25

${ }^{16} \mathrm{http}: / /$ sci.esa.int/science-e/www/area/index.cfm?fareaid=100
} 


\section{Imprints on the CMB at small scales: perspectives from the SKA}

In the view of the extreme resolution of the SKA, it is natural to expect that it will provide a particular improvement for our understanding of astrophysical processes able to produce SZ effect or other signatures in the CMB at very small angular scales. I will report here on three specific topics, from lower to higher redshifts, of particular interest for extragalactic astrophysics and cosmology, properly revised in the context of the SKA scientific case [13] and updated in some cases to account for WMAP 3-yr results and recent analyses.

\subsection{Thermal SZ effect at galaxy scale}

The proto-galactic gas is expected to have a large thermal energy content, leading to a detectable SZ signal, both when the protogalaxy collapses with the gas shock-heated to the virial temperature [77, 98], and in a later phase as the result of strong feedback from a flaring active nucleus (see slide 83; see, e.g., [41, 59, 60, 1, 72, 45]. The astrophysical implications of these scenarios have been investigated by [25].

A fully ionized gas with a thermal energy density $\varepsilon_{\text {gas }}$ within the virial radius

$$
R_{\mathrm{vir}}=\left(\frac{3 M_{\mathrm{vir}}}{4 \pi \rho_{\mathrm{vir}}}\right)^{1 / 3} \simeq 1.610^{2} h^{-2 / 3}\left(1+z_{\mathrm{vir}}\right)^{-1}\left(\frac{M_{\mathrm{vir}}}{10^{12} M_{\odot}}\right)^{1 / 3} \mathrm{kpc}
$$

transfers to the CMB an amount $\Delta \varepsilon \simeq\left(\varepsilon_{\mathrm{gas}} / t_{C}\right) 2\left(R_{\mathrm{vir}} / c\right)$ of thermal energy through Thomson scattering producing a Comptonization parameter [101] $y \simeq(1 / 4) \Delta \varepsilon / \varepsilon_{\mathrm{CMB}}$ (see slide 84). Here $\rho_{\text {vir }} \simeq 200 \rho_{u}, \rho_{u}=1.88 \times 10^{-29} h^{2}\left(1+z_{\mathrm{vir}}\right)^{3} \mathrm{~g} \mathrm{~cm}^{-3}$ is the mean density of the universe at the virialization redshift $z_{\mathrm{vir}}$.

Assuming the binding energy $\left(E_{\mathrm{b}, \mathrm{gas}}=M_{\mathrm{gas}} v_{\mathrm{vir}}^{2}, v_{\mathrm{vir}}=162 h^{1 / 3}(1+z)^{1 / 2}\left(M_{\mathrm{vir}} / 10^{12} M_{\odot}\right)^{1 / 3}\right.$ $\mathrm{km} \mathrm{s}^{-1}$ being the circular velocity of the galaxy at its virial radius $\left.[61,10]\right)$ to characterize the thermal energy content of the gas, $E_{\text {gas }}$, the amplitude of the SZ dip observable in the RJ region ${ }^{17}$ can be written as (see slide 85):

$$
|\Delta T|_{\mathrm{RJ}}=2 y T_{0} \simeq 1.7\left(\frac{h}{0.5}\right)^{2}\left(\frac{1+z_{\mathrm{vir}}}{3.5}\right)^{3} \frac{M_{\mathrm{gas}} / M_{\mathrm{vir}}}{0.1} \frac{M_{\mathrm{vir}}}{10^{12} M_{\odot}} \frac{E_{\mathrm{gas}}}{E_{\mathrm{b}, \mathrm{gas}}} \mu \mathrm{K} .
$$

This SZ effect shows up on small (typically sub-arcmin) angular scales.

Quasar-driven blast-waves could inject into the ISM an amount of energy several times higher than the gas binding energy, thus producing larger, if much rarer, SZ signals. A black-hole (BH) accreting a mass $M_{\mathrm{BH}}$ with a mass to radiation conversion efficiency $\varepsilon_{\mathrm{BH}}$ releases an energy $E_{\mathrm{BH}}=$ $\varepsilon_{\mathrm{BH}} M_{\mathrm{BH}} c^{2}$. The standard value for the efficiency $\varepsilon_{\mathrm{BH}}=0.1$ and a fraction $f_{h}=0.1$ for the energy fed in kinetic form and generating strong shocks turning it into heat (see slide 85) are assumed here for numerical estimates.

\footnotetext{
${ }^{17}$ Note that $|\Delta T|_{\mathrm{RJ}} \simeq 2 u T_{0}$ or $|\Delta T|_{\mathrm{RJ}} \simeq 3 u T_{0}$ respectively when $\Delta T_{\mathrm{RJ}}=T_{\mathrm{RJ}}-T_{i, 0}$ or $\Delta T_{\mathrm{RJ}}=T_{\mathrm{RJ}}-T_{0}$ is considered (see slide 17), being $T_{i, 0}<T_{0}$ in the presence of a global heating process. This consideration applies to the case of CMB global Comptonization distortions. In this context, since we are considering a (local) SZ effect characterized by a Comptonization parameter $y$, with fully negligible global energy exchange, $|\Delta T|_{\mathrm{RJ}} \simeq 2 y T_{0}$.
} 
Using the recent re-assessment by [94] of the well known correlation between the BH mass and the stellar velocity dispersion, $M_{\mathrm{BH}}=1.4 \times 10^{8}(\sigma / 200 \mathrm{~km} / \mathrm{s})^{4} \mathrm{M}_{\odot}$, one gets

$$
\frac{E_{\mathrm{BH}}}{E_{\mathrm{b}, \mathrm{gas}}} \simeq 4.7\left(\frac{h}{0.5}\right)^{-2 / 3} \frac{\varepsilon_{\mathrm{BH}}}{0.1} \frac{f_{h}}{0.1} \frac{1+z_{\mathrm{vir}}}{3.5}\left(\frac{M_{\mathrm{gas}} / M_{\mathrm{vir}}}{0.1}\right)^{-1}\left(\frac{M_{\mathrm{vir}}}{10^{12} M_{\odot}}\right)^{-1 / 3} .
$$

The amplitude of the SZ dip in the RJ region due to quasar heating of the gas is then estimated as (see slide 86):

$$
\left|\left(\frac{\Delta T}{T}\right)_{\mathrm{RJ}}\right| \simeq 1.8 \times 10^{-5} \frac{f_{h}}{0.1}\left(\frac{h}{0.5}\right)^{2}\left(\frac{\varepsilon_{\mathrm{BH}}}{0.1}\right)^{1 / 2}\left(\frac{E_{\mathrm{BH}}}{10^{62}}\right)^{1 / 2}\left(\frac{1+z}{3.5}\right)^{3} .
$$

Following [72], an isothermal density profile of the galaxy is adopted. The virial radius, encompassing a mean density of $200 \rho_{u}$, is then:

$$
R_{\mathrm{vir}} \simeq 120\left(\frac{h}{0.5}\right)^{-1}\left(\frac{E_{\mathrm{BH}}}{10^{62}}\right)^{1 / 4}\left(\frac{\varepsilon_{\mathrm{BH}}}{0.1}\right)^{-1 / 4}\left(\frac{1+z_{\mathrm{vir}}}{3.5}\right)^{-3 / 2} \mathrm{kpc},
$$

corresponding to an angular radius:

$$
\theta_{S Z} \simeq 17^{\prime \prime}\left(\frac{E_{\mathrm{BH}}}{10^{62}}\right)^{1 / 4}\left(\frac{\varepsilon_{\mathrm{BH}}}{0.1}\right)^{-1 / 4}\left(\frac{1+z_{\mathrm{vir}}}{3.5}\right)^{-3 / 2} \frac{d_{A}(2.5)}{d_{A}(z)}
$$

where $d_{A}(z)$ is the angular diameter distance.

The angular scales of these SZ signals from galaxies are of the order of $\approx 10^{\prime \prime}$, then of particular interest for a detailed mapping with the SKA and XEUS in the radio and X-ray, respectively (see slide 87). The probability of observing these SZ sources on a given sky field at a certain flux detection level and the corresponding fluctuations are mainly determined by the redshift dependent source number density $\phi_{\mathrm{SZ}}\left(S_{\mathrm{SZ}}, z\right)$ per unit interval of the SZ (decrement) flux $S_{\mathrm{SZ}}$. The lifetime of the considered SZ sources is crucial to determine their number density (see slide 88 ).

For quasar-driven blast-waves the lifetime of the active phase, $t_{\mathrm{SZ}}$, is approximately equal to the time for the shock to reach the outer boundary of the host galaxy. Assuming a self-similar blast-wave expanding in a medium with an isothermal density profile, $\rho \propto r^{-2}$, one gets:

$$
t_{\mathrm{SZ}} \simeq 1.5 \times 10^{8}\left(\frac{h}{0.5}\right)^{-3 / 2}\left(\frac{E_{\mathrm{BH}}}{10^{62} \mathrm{erg}}\right)^{1 / 8}\left(\frac{\varepsilon_{\mathrm{BH}}}{0.1}\right)^{-5 / 8}\left(\frac{f_{h}}{0.1}\right)^{-1 / 2}\left(\frac{1+z}{3.5}\right)^{-9 / 4} \mathrm{yr} .
$$

The evolving B-band luminosity function of quasars, $\phi\left(L_{B}, z\right)$, (see e.g. [70]) can be then adopted to derive the source number density $\phi_{\mathrm{SZ}}\left(S_{\mathrm{SZ}}, z\right)$ according to

$$
\phi_{\mathrm{SZ}}\left(S_{\mathrm{SZ}}, z\right)=\phi\left(L_{B}, z\right) \frac{t_{\mathrm{SZ}}}{t_{\mathrm{q}, \mathrm{opt}}} \frac{d L_{B}}{d S_{\mathrm{SZ}}}
$$

where $L_{B}\left(S_{\mathrm{SZ}}, z\right)$ is the blue luminosity of a quasar at redshift $z$ causing a (negative) SZ flux $S_{\mathrm{SZ}}$, and $t_{\mathrm{q}, \mathrm{opt}}$ is the duration of the optically bright phase of the quasar evolution (see slide 89).

For the proto-galactic gas $t_{\mathrm{SZ}}$ should be replaced by the gas cooling time, $t_{\mathrm{cool}}$. Assuming that quasars can be used as effective signposts for massive spheroidal galaxies in their early evolutionary phases [35] and that they emit at the Eddington limit and using the relation by [30] between 
the mass of the dark-matter halo, $M_{\mathrm{vir}}$, and the mass of the central black-hole, $M_{\mathrm{BH}} / 10^{8} M_{\odot} \sim$ $0.1\left(M_{\mathrm{vir}} / 10^{12} M_{\odot}\right)^{1.65}$, the number density of sources with gas at virial temperature can be straightforwardly related to the quasar luminosity function $\phi\left(L_{B}, z\right)$ (see slide 90 ).

In spite of the many uncertainties of these models, it is remarkable that the CMB fluctuations (dominated at small scales by the Poisson contribution) induced by the SZ effect of these source populations could contribute to the CBI [54] anisotropy measure and, in particular, could contribute to explain part of the excess in the angular power spectrum found by BIMA [24] at multipoles $\ell \approx(4-10) \times 10^{3}$ (see slide 91 ), a certain fraction of it being likely produced by high-redshift dusty galaxies, whose fluctuations may be strongly enhanced by the effect of clustering [93].

Also, the integrated Comptonization distortion produced by quasar-driven blast-waves has been computed by [72] through a quite general "energetic" approach, not particularly related to a detailed physical assumption: they found $u \sim 2.4 \times 10^{-6}$.

A direct probe of these models and, possibly, their accurate knowledge through a precise high resolution imaging is then of particular interest. Slide 92 shows the number counts at $20 \mathrm{GHz}$ predicted by these models: in a single SKA FOV about few $\times 10^{2}-10^{3} \mathrm{SZ}$ sources with fluxes above $\sim 100$ nJy could be then observed in few hours of integration. Given the typical source sizes, we expect a blend of sources in the SKA FOV at these sensitivity levels, while much shorter integration times, $\sim$ sec, on many FOV would allow to obtain much larger maps with a significant smaller number of resolved SZ sources per FOV. Both surveys on relatively wide sky areas and deep exposures on limited numbers of FOV are interesting and easily obtainable with SKA (see slide 93).

A different scenario to jointly explain the power excess found by BIMA and the high redshift reionization detected by WMAP 1-yr data [50] $\left(z_{\text {reion }} \sim 15-20\right)$ was proposed by [64] (see slide 94). It involves hot gas winds powered by pair-instability supernovae (SN) explosions from the first generation of very massive stars at very low metallicity able to photoevaporate the gas in the halo potential. The SN remnants should then dissipate their energy in the intergalactic medium (IGM) and about 30-100\% of their energy would be transferred to the CMB via Compton cooling. However, the resulting SZ effect from individual sources is estimated to be too faint (corresponding to $\approx \mathrm{few} \times 10^{-2} \mathrm{nJy}$ ) to be observable even by SKA. On the contrary, the resulting SZ effect from these sources could be relevant in statistical sense. It is claimed to explain the high $\ell$ BIMA excess of the $\mathrm{CMB}$ angular power spectrum and to be able to generate a global Comptonization distortion parameter $u \sim$ few $\times 10^{-6}$ (on the other hand, a certain decrease of its effect with respect to this picture could be expected in the light of the lowering of the reionization redshift as revised by WMAP 3-yr data).

\subsection{Free-free emitters}

The understanding of the ionizing emissivity of collapsed objects and the degree of gas clumping is crucial for reionization models. The observation of diffuse gas and Population III objects in thermal bremsstrahlung as a direct probe of these quantities has been investigated by [63]. Freefree emission produces both global and localized spectral distortion of the CMB (see slide 95). A natural way to distinguish between free-free distortion by ionized halos rather than by diffuse ionized IGM is represented by observations at high resolution of dedicated sky areas and by the fluctuations in the free-free background. In the model by [63] halos collapse and form a starburst 
lasting $t_{o}=10^{7} \mathrm{yr}$, then recombine and no longer contribute to the free-free background. By adopting a Press-Schechter model [74, 7] for the number density of collapsed halos per mass interval, $d n_{P S} / d M$, [63] exploited the expression by [84] for the collapse rate of halos per mass interval per unit comoving volume (see slide 96):

$$
\frac{d \dot{N}^{f o r m}}{d M}(M, z)=\frac{1}{D} \frac{d D}{d t} \frac{d n_{P S}}{d M}(M, z) \frac{\delta_{c}^{2}}{\sigma^{2}(M) D^{2}}
$$

here $D(z)$ is the growth factor and $\delta_{c}=1.7$ is the threshold above which mass fluctuations collapse. The expected comoving number density of ionized halos in a given flux interval as a function of redshift

$$
\frac{d N_{\text {halo }}}{d S d V}(S, z)=\int_{t(z)}^{t(z)-t_{o}} d t \frac{d \dot{N}^{f o r m}}{d M} \frac{d M}{d S}
$$

can be then computed given the expected flux from a halo of mass $\mathrm{M}$ at redshift $z, S=S(M, z)$, and the starburst duration, $t_{o}$. Adopting a cut-off mass for a halo (see slide 97) to be ionized of $M_{*}=10^{8}(1+z / 10)^{-3 / 2} M_{\odot}$ (the critical mass needed to attain a virial temperature of $10^{4} \mathrm{~K}$ to excite atomic hydrogen cooling), [63] computed the number counts of sources above the flux limit $S_{c}$ from the zeroth moment of the intensity distribution moments due to sources above a redshift $z_{\min }$,

$$
\left\langle S^{n}\left(>z_{\min }, S_{c}\right)\right\rangle=\int_{z_{\min }}^{\infty} d z \int_{S_{\min }(z)}^{S_{\max }} d S \frac{d N_{\mathrm{halo}}}{d S d V} \frac{d V}{d z d \Omega} S^{n}
$$

by setting $S_{\max } \rightarrow \infty$ and $S_{\min }(z)=\max \left(S_{c}, S_{*}(z)\right)$, where $S_{*}(z)$ denotes the flux from a halo of minimum mass $M_{*}$ at redshift $z$.

The relation $\dot{N}_{\text {recomb }}=\alpha_{B}\left\langle n_{e}^{2}\right\rangle V \approx\left(1-f_{\text {esc }}\right) \dot{N}_{\text {ion }}$, between the production rate of recombination line photons, $\dot{N}_{\text {recomb }}$, and the production rate of ionizing photons, $\dot{N}_{\text {ion }}$, (here $\alpha_{B}$ is the recombination coefficient and $f_{\text {esc }}(\approx$ some $\%)$ is the escape fraction for ionizing photons) implies that the source luminosities in $\mathrm{H} \alpha$ and free-free emission $\left(\propto n_{e}^{2} V\right)$ are proportional to the production rate of ionizing photons (see slide 98). Over a wide range of nebulosity conditions [40] found that $\simeq 0.45 \mathrm{H} \alpha$ photons are emitted per Lyman continuum photon; thus $\mathrm{L}(\mathrm{H} \alpha)=$ $1.4 \times 10^{41} \dot{N}_{\text {ion }} /\left(10^{53} \mathrm{ph} \mathrm{s}^{-1}\right) \mathrm{erg} \mathrm{s}^{-1}$. Given the free-free volume emissivity [80] in the case of an approximate mild temperature dependence with a power law (a velocity averaged Gaunt factor $\bar{g}_{f f}=4.7$ is assumed), $\varepsilon_{v}=3.2 \times 10^{-39} n_{e}^{2}\left(T / 10^{4} K\right)^{-0.35} \mathrm{erg} \mathrm{s}^{-1} \mathrm{~cm}^{-3} \mathrm{~Hz}^{-1}$, it is found

$$
L_{v}^{f f}=1.2 \times 10^{27} \frac{\dot{N}_{\text {ion }}}{10^{53} \mathrm{phs}^{-1}} \mathrm{erg} \mathrm{s}^{-1} \mathrm{~Hz}^{-1} .
$$

Assuming the starburst model of [37] normalised to the observed metallicity $10^{-3} Z_{\odot} \leq Z \leq 10^{-2} Z_{\odot}$ of the IGM at $z \sim 3$ (resulting into a constant fraction of the gas mass turning into stars, $1.7 \% \leq$ $f_{\text {star }} \leq 17 \%$, in a starburst which fades after $\sim 10^{7} \mathrm{yr}$ ), [63] derived a production rate of ionizing photons as a function of halo mass given by (see slide 99 ):

$$
\dot{N}_{\text {ion }}(M)=2 \times 10^{53} \frac{f_{\text {star }}}{0.17} \frac{M}{10^{9} M_{\odot}} \mathrm{ph} \mathrm{s}^{-1}
$$


which specifies the above free-free ionized halo luminosity. The corresponding flux is then:

$$
S_{\mathrm{ff}}=\frac{L_{v}^{\mathrm{ff}}}{4 \pi d_{L}^{2}}(1+z) \approx 2.5\left(\frac{1+z}{10}\right)^{-1} \frac{\mathrm{M}}{10^{9} \mathrm{M}_{\odot}}\left(\frac{\mathrm{T}}{10^{4} \mathrm{~K}}\right)^{-0.35} \mathrm{nJy} .
$$

Clearly, SKA will allow to detect only bright sources with deep exposures. The ionized halo number counts can be calculated from Eq. (4.9). The result by [63] is reported in slide 100 (and compared with [68]): SKA should be able to detect $\sim 10^{4}$ individual free-free emission sources with $z>5$ in 1 square degree above a source detection threshold of $70 \mathrm{nJy}$. The redshift information from the Balmer line emission detectable by the Next Generation Space Telescope (NGST) can be used to discriminate ionized halos from other classes of radio sources.

Ionized halos may contribute to the temperature fluctuations. In particular, the Poisson contribution is predicted to be larger (smaller) than the clustering one at scales smaller (larger) than $\sim 30^{\prime \prime}$ [63]. On the other hand, both are likely dominated by the radio source contribution.

Finally, the integrated emission from ionized halos produces a global CMB spectral distortion, $\Delta T_{f f}=c^{2}\langle S\rangle / 2 k_{B} v^{2}$, that can be computed from the mean sky averaged signal $\langle S\rangle$ (see slide 101). By using Eq. (4.9) (since no point source removal is feasible at degree scales) with $z_{\min }$ and $S_{c}=$ 0 , [63] found a free-free distortion $\Delta T_{f f}=3.4 \times 10^{-3} \mathrm{~K}$ at $2 \mathrm{GHz}$, corresponding to a free-free distortion parameter $y_{B} \simeq 1.5 \times 10^{-6}$, well within the observational capability of a next generation of CMB spectrum experiments at long wavelengths (see next section).

\subsection{SKA contribution to future CMB spectrum experiments}

The current limits on CMB spectral distortions and the constraints on energy dissipation processes $\left|\Delta \varepsilon / \varepsilon_{i}\right| \lesssim 10^{-4}$ in the plasma [81] are mainly set by COBE/FIRAS [56, 31] (see slides 102-104). CMB spectrum experiments from space, DIMES [48] (see also [49]) at $\lambda \gtrsim 1 \mathrm{~cm}$ and FIRAS II [32] at $\lambda \lesssim 1 \mathrm{~cm}$, have been proposed with an accuracy potentially able to constrain (or probably detect) energy exchanges 10-100 times smaller than the FIRAS upper limits (see slide 105). Also, a promising opportunity in this respect is represented by the recent renaissance of interest for the Moon as a base for extremely accurate observations of the universe. Slides 111-113 display some of the fundamental ideas for a CMB spectrum experiment at long wavelengths, proposed in a recent study for ASI, that forsee an ultimate huge experiment at centimetre and decimetre wavelengths anticipated by precursor experiments at centimetre wavelengths, able to significantly improve the current observational scenario [17].

Long wavelength experiments may probe in particular dissipation processes at early times $\left(z \gtrsim 10^{5}\right)$ resulting in Bose-Einstein like distortions [90, 23, 11] and free-free distortions [3] possibly generated by heating (but, although disfavoured by WMAP, in principle also by cooling [88]) mechanisms at late epochs $\left(z \lesssim 10^{4}\right)$, before or after the recombination era [16].

Typical shapes of distorted spectra potentially detectable with new experiments are shown in slide 108 while slides 109 and 110 display the possible improvements in our understanding of the energy exchange in the primeval plasma respectively in the absence and in the presence of detection of spectral distortions. To firmly observe such small distortions the Galactic and extragalactic foreground contribution should be accurately modelled and subtracted (see slide 114). Recent progress on radio source counts at $1.4 \mathrm{GHz}$ have been presented in [73]. On the other hand, the very faint tail of radio source counts is essentially unexplored and their contribution to the radio 
background at very low brightness temperature is not accurately known (see slide 114). For illustration, by assuming differential source number counts, $N(S)$, given by $\log N(S) / \Delta N_{0} \sim a \log S+b$, with $\Delta N_{0} \sim 150 S^{-2.5} \mathrm{sr}^{-1} \mathrm{Jy}^{-1}$ ( $S$ in Jy) [92], for $a \sim 0.4-0.6$ and $b \sim-(0.5-1)$, one derives a contribution to the radio background at $5 \mathrm{GHz}$ from sources between $\sim 1 \mathrm{nJy}$ and $\sim 1 \mu \mathrm{Jy}$ between few tens of $\mu \mathrm{K}$ and few $\mathrm{mK}$ (see slide 115). These signals are clearly negligible compared to the accuracy of current CMB spectrum experiments, in particular at $\lambda \gtrsim 1 \mathrm{~cm}$, but are significant at the accuracy level on CMB distortion parameters potentially achievable with novel experiments at long wavelengths. This effect is small compared to the Galactic radio emission, whose accurate knowledge currently represents the major astrophysical problem in CMB spectrum experiments, but, differently from Galactic emission, it is isotropic at the angular scales of few degrees and can not be then subtracted from the $\mathrm{CMB}$ monopole temperature on the basis of its angular correlation properties. With accurate absolute measures on a wide frequency coverage a fit including both CMB distorted spectra and astrophysical contributions can be searched (see [81] for an application to FIRAS data) but a direct radio background estimate from precise number counts will certainly improve the robustness of this kind of analyses.

The SKA sensitivity at $20 \mathrm{GHz}$ will allow the detection (to $5 \sigma$ ) of sources down to a flux level of $\simeq 200 \mathrm{nJy}(\simeq 60,20,6 \mathrm{nJy})$ in $1\left(10,10^{2}, 10^{3}\right)$ hour(s) of integration over the $\simeq 1$ mas $(\mathrm{FWHM})$ resolution element; similar numbers (from $\simeq 250$ to $8 \mathrm{nJy}$ in an integration time from 1 to $10^{3}$ hours, respectively) but on a resolution element about 10 times larger will be reached at $\approx \mathrm{GHz}$ frequencies by using a frequency bandwidth of about $25 \%$.

Therefore, the SKA accurate determination of source number counts down to very faint fluxes can directly help the solution of one fundamental problem of the future generation of CMB spectrum space experiments at $\lambda \gtrsim 1 \mathrm{~cm}$ (see slide 116).

\section{Conclusion}

The thermal plasma in the intergalactic and intracluster medium and at galactic scales leaves imprints on the CMB through the Thomson scattering of CMB photons on hot electrons (SZ effect) and the free-free emission. Since its original formulation, the SZ effect, first elaborated for clusters of galaxies, has been recognized as a "powerful laboratory" for our comprehension of physical processes in cosmic structures and to derive crucial information on some general properties of the universe.

Many thousands of galaxy clusters can be identified by XMM, Planck, and SDSS. SKA will allow to map the thermal and density structure of clusters of galaxies at radio and centimetre bands with unprecedented resolution and sensitivity and with an extremely accurate control of extragalactic radio source contamination. The signatures from SZ effects and free-free emission at galactic scales and in the intergalactic medium probe the structure evolution at various cosmic times. The detection of these sources and their imaging at the high resolution and sensitivity achievable with SKA will greatly contribute to the comprehension of crucial cosmological and astrophysical aspects, as the physical conditions of early ionized halos, quasars and proto-galactic gas. A wealth of information will be available by combining SKA observations with those expected at higher frequencies by ALMA and NGST and with the high quality X-ray data promised by XEUS. Also, the spectacular improvement in our understanding of the properties of extragalactic radio sources 
at very faint fluxes achievable with SKA will allow to accurately model their contribution to the diffuse background from radio to centimetre wavelengths and will greatly contribute to the interpretation of next generation of CMB spectrum experiments.

In conclusion, although not specifically devoted to CMB studies, because of its high resolution and the limited high frequency coverage, the extreme sensitivity and resolution of SKA may be fruitfully used for a detailed mapping of the thermal plasma properties in the intergalactic and intracluster medium and at galaxy scale and to probe the thermal plasma history at early times.

Acknowledgements - I warmly thank G. De Zotti and L. Feretti for numberless and constructive conversations and for the collaboration to the SKA scientific case. It it a pleasure to thank the School organizers for the kind invitation and generous and helpful logistic support. The use of the CMBFAST $\operatorname{code}^{18}$ (see e.g. [83]) is acknowledged.

\section{References}

[1] Aghanim N., Balland C., Silk, J., 2000, A\&A, 357, 1

[2] Arnaud M., 2005, in Proc. Int. School of Physics "E. Fermi”, Course CLIX, Background Microwave Radiation and Intracluster Cosmology, eds. Melchiorri F. \& Rephaeli Y., IOS Press, pg. 77

[3] Barlett J.G., Stebbins A., 1991, ApJ, 371, 8

[4] Bennett C.L., et al., 2003, ApJS, 148, 1

[5] Benson B.A., et al., 2003, ApJ, 592, 674

[6] Bersanelli M., Maino D., Mennella A., 2002, Riv. Nuovo Cimento, 25(9), 1

[7] Bond J.R., Cole S., Efstathiou G., Kaiser N., 1991, ApJ, 379, 440

[8] Bond J.R., Crittenden R., Davis R.L., Efstathiou G., Steinhardt P.J., 1994, PRL, 72, 13

[9] Bond J.R., Davis R.L., Steinhardt P.J., 1995, Astroph. Lett. and Comm., 32, 53

[10] Bullock J.S., et al., 2001, MNRAS, 321, 559

[11] Burigana C., Danese L., De Zotti G., 1991, A\&A, 246, 59

[12] Burigana C., De Zotti G., Danese L., 1995, A\&A, 303, 323

[13] Burigana C., De Zotti G., Feretti L., 2004, New Astronomy Reviews, 48, 1107

[14] Burigana C., Finelli F., Salvaterra R., Popa L.A., Mandolesi N., 2004, in Recent Research Developments in Astronomy \& Astrophysics - Vol. 2, 59

[15] Burigana C., Popa L.A., Salvaterra R., Schneider R., Choudhury T.R., Ferrara A., 2007, MNRAS, in press, astro-ph/0712.1913

[16] Burigana C., Salvaterra R., 2003, MNRAS, 342, 543

[17] Burigana C., et al., 2007, Perspectives for future experiments and studies on cosmic background radiation from the Moon, in Italian Vision for Moon Exploration - Observation of the Universe from the Moon, ASI Contract I/032/06/04, pg. 125

\footnotetext{
${ }^{18}$ http://www.cmbfast.org/
} 
[18] Carlstrom J.E., Holder G.P., Reese E.D., 2002, ARAA, 40, 643

[19] Cavaliere A., Danese L., De Zotti G., 1978, in COSPAR, Plenary Meeting, 21st, Innsbruck, Austria, May 29-June 10, 1978

[20] Cavaliere A., Danese L., De Zotti G., 1979, A\&A, 75, 322

[21] Ciardi B., 2007, Lecture at this School

[22] Danese L., De Zotti G., 1977, Riv. Nuovo Cimento, 7, 277

[23] Danese L., De Zotti G., 1980, A\&A, 84, 364

[24] Dawson K.S., et al., 2002, ApJ, 581, 86

[25] De Zotti G., et al., 2004, in Proc. Int. Symp. Plasmas in the Laboratory and in the Universe: new insights and new challenges, Como, September 2003, eds. Bertin G., Farina D., Pozzoli R., pg. 375, astro-ph/0401191

[26] da Silva A.C., Kay S.T., Liddle A.R., Thomas P.A., Pearce F.R., Barbosa D., 2001, ApJ, 561, L15

[27] Dolag K., Hansen F.K., Roncarelli M., Moscardini L., 2005, MNRAS, 363, 29

[28] Efstathiou G., Bond J.R., 1999, MNRAS, 304, 75

[29] Fabbri R., 1981, Ap. Space Sci., 77, 529

[30] Ferrarese L., 2002, in Current high-energy emission around black holes, eds. C.-H. Lee \& H.-Y. Chang, Singapore: World Scientific Pub.

[31] Fixsen D.J., Cheng E.S., Gales J.M., Mather J.C., Shafer R.A., Wright E.L., 1996, ApJ, 473, 576

[32] Fixsen D.J., Mather J.C., 2002, ApJ, 581, 817

[33] Gnedin N.Y., Jaffe A.H., 2001, ApJ, 551, 3

[34] Gould R.J., 1984, ApJ, 285, 275

[35] Granato G.L., et al., 2001, MNRAS, 324, 757

[36] Gunn J.E., 1978, in Observational Cosmology, eds. Maeder A. et al., Geneva Observatory

[37] Haiman Z., Loeb A., 1998, ApJ, 503, 505

[38] Hansen F.K., Branchini E., Mazzotta P., Cabella P., Dolag K., 2005, MNRAS, 361, 753

[39] Hall P., 2007, Lecture at this School

[40] Hummer D.G., Storey P.J., 1987, MNRAS, 224, 801

[41] Ikeuchi S., 1981, PASJ, 33, 211

[42] Itoh N., 2005, in Proc. Int. School of Physics “E. Fermi”, Course CLIX, Background Microwave Radiation and Intracluster Cosmology, eds. Melchiorri F. \& Rephaeli Y., IOS Press, pg. 295

[43] Jarosik N., et al., 2007, ApJS, 170, 263

[44] Jones M.E., 2003, in The scientific promise of the Square Kilometer Array, SKA Workshop, Oxford, 7 November 2002, eds. Kramer M., Rawlings S., pg. 47, http://www.skatelescope.org/documents/ Workshop_Oxford2002.pdf

[45] Lapi A., Cavaliere A., De Zotti G., 2003, ApJ, 597, L93

[46] Karzas W., Latter R., 1961, ApJS, 6, 167 
[47] Knox L., 1995, Phys. Rev. D., 48, 3502

[48] Kogut A., 1996, Diffuse Microwave Emission Survey, in XVI Moriond Astrophysics meeting Microwave Background Anisotropies, March 16-23, Les Arcs, France, astro-ph/9607100

[49] Kogut A., 2003, New Astronomy Reviews, 47, 945

[50] Kogut A., et al., 2003, ApJS, 148, 161

[51] Kompaneets A.S., 1956, Zh.E.T.F., 31, 876 [Sov. Phys. JETP, 4, 730 (1957)]

[52] Ma C.-P., Fry J.N., 2002, PRL, 88, 211301

[53] Mandolesi N., et al., 1998, Planck LFI, A proposal submited to the ESA

[54] Mason B.S., et al., 2003, ApJ, 591, 540

[55] Massardi M., De Zotti G., 2004, A\&A, 424, 409

[56] Mather J.C., et al., 1990, ApJ, 354, L37

[57] Mather J.C., Fixsen D.J., Shafer R.A., Mosier C., Wilkinson D.T., 1999, ApJ, 512, 511

[58] Melchiorri F., Rephaeli Y., 2005, Proc. Int. School of Physics “E. Fermi”, Course CLIX, Background Microwave Radiation and Intracluster Cosmology, eds. Melchiorri F. \& Rephaeli Y., IOS Press

[59] Natarajan P., Sigurdsson S., Silk, J., 1998, MNRAS, 298, 577

[60] Natarajan P., Sigurdsson S., 1999, MNRAS, 302, 288

[61] Navarro J.F., Frenk C.S., White S.D.M., 1997, ApJ, 490, 393

[62] Norman M.L., 2005, in Proc. Int. School of Physics “E. Fermi”, Course CLIX, Background Microwave Radiation and Intracluster Cosmology, eds. Melchiorri F. \& Rephaeli Y., IOS Press, pg. 1

[63] Oh S.P., 1999, ApJ, 527, 16

[64] Oh S.P., Cooray A., Kamionkowski M., 2003, MNRAS, 342, L20

[65] Ostriker J.P., Vishniac E.T., 1986, ApJ, 306, L51

[66] Page L., et al., 2007, ApJS, 170, 335

[67] Partridge R.B., 1995, 3K: The Cosmic Microwave Background Radiation, Camdridge University Press

[68] Partridge R.B., Richards E.A., Fomalont E.B., Kllerman K.I., Windhorst R., 1997 ApJ, 483, 38

[69] Peebles P.J.E., 1993, Principles of Physical Cosmology, Princeton University Press

[70] Pei Y.,1995, ApJ, 438, 623

[71] Peyraud J., 1968, J. Phys., 29, 306

[72] Platania P., Burigana C., De Zotti G., Lazzaro E., Bersanelli M., 2002, MNRAS, 337, 242

[73] Prandoni I., et al., 2001, A\&A, 365, 392

[74] Press W.H., Schechter P., 1974, ApJ, 187, 452

[75] Planck Collaboration, 2006, The Scientific Programme of Planck, astro-ph/0604069

[76] Puget J.L., et al., 1998, HFI for the Planck Mission, A proposal submited to the ESA

[77] Rees M.J., Ostriker J.P., 1977, MNRAS, 179, 541

[78] Rephaeli Y., 1995, ARA\&A, 33, 541 
[79] Rephaeli Y., Sadeh S., Shimon M., 2005, in Proc. Int. School of Physics “E. Fermi”, Course CLIX, Background Microwave Radiation and Intracluster Cosmology, eds. Melchiorri F. \& Rephaeli Y., IOS Press, pg. 57

[80] Rybicki G.B., Lightman A.P., 1979, Radiative Processes in Astrophysics, Wiley, New York

[81] Salvaterra R., Burigana C., 2002, MNRAS, 336, 592

[82] Schneider R., Salvaterra R., Choudhury T.R., Ferrara A., Burigana C., Popa L.A., 2007, MNRAS, in press, astro-ph/0712.0538

[83] Seljak U., Zaldarriaga M., 1996, ApJ, 469, 437

[84] Sasaki S., 1994, PASJ, 46, 427

[85] Silk J., 1968, ApJ, 151, 459

[86] Silk J., White S.D.M., 1978, ApJ, 226, L103

[87] Springel V., White M., Hernquist L., 2001, ApJ, 549, 681

[88] Stebbins A., Silk J., 1986, ApJ, 169, 1

[89] Subrahmanyan R., Ekers R.D., 2002, in the XXVIIth General Assembly of the URSI, August 17-24, Maastricht, The Netherlands, astro-ph/0209569

[90] Sunyaev R.A., Zeldovich Ya.B., 1970, Ap\&SS, 7, 20

[91] Sunyaev R.A., Zeldovich Ya.B., 1972, Comm. Astrophys. Space Phys., 4, 173

[92] Toffolatti L., et al., 1998, MNRAS, 297, 117

[93] Toffolatti L., et al., 2005, A\&A, 438, 475

[94] Tremaine S., et al., 2002, ApJ, 574, 740

[95] Tricomi F.G., 1957, Equazioni a Derivate Parziali, ed. Cremonese, Roma

[96] Vishniac E.T., 1987, ApJ, 322, 597

[97] Weinberg S., 1972, Gravitation and Cosmology, J. Wiley, New York

[98] White S.D.M., Rees M.J., 1978, MNRAS, 183, 341

[99] Wright E.L., 1979, ApJ, 232, 348

[100] Zeldovich Ya.B., Levich E.V., 1968, Zh. Eksp. Teor. Fiz., 55, 2423 [Sov. Phys. JEPT, 28, 1267 (1969)]

[101] Zeldovich Ya.B., Sunyaev R.A., 1969, Ap\&SS, 4, 301

[102] Zeldovich Ya.B., Illarionov A.F., Sunyaev R.A., 1972, Zh. Eksp. Teor. Fiz., 62, 1216 [Sov. Phys. JEPT, 35, 643]

[103] Zizzo A., Burigana C., 2005, NA, 11, 1 